have been proposed to play a role in the different incidence of osteoporotic hip fractures among different countries.

Objectives Our objective consisted of studying the normal distribution of HAL in the Portuguese population and try to identify clinical parameters capable of predicting HAL, therefore reinforcing indication of bone densitometry.

Methods A randomly selected sample of 1705 inhabitants from the centre of Portugal, (474 males; 1231 females), aged 19 to 89 were included in the study. HAL was evaluated by DEXA (Hologic) using inbuilt software. Patients were asked and examined for age, present height and weight and at age 25, age at menarche and arm span. Following correlation studies, significant variables were included in stepwise regression analysis (SPSS).

Results HAL was significantly longer in males than in females $(12,44 \pm 0,69$ vs $10,84 \pm 0,69 ; \mathrm{p}<0.001)$. In men, significant correlations $(\mathrm{p}<0.001)$ were found between HAL and height at age 25 (r:0.483), weight at age 25 (r:0.176) and arm span ( $\mathrm{r}: 0.0272)$. Using stepwise regression only height at age 25 was retained as significant (Model r: 0.490; Adj. R2: 0,238 - p < $0.001)$. In women, age ( $\mathrm{r}: 0,06)$, height at $25(0,304)$, present height $(0,314)$, weight at $25(0,214)$, present weight $(0,058)$ and arm span $(0,267)$, all showed significant correlations. Stepwise regression showed significant contributions for armspan, height and weight at age 25 . The best fitting model explained $16,5 \%$ of the variance. In our population no major difference in HAL has been observed between the young (20-30 years old) and the elderly $(>70)$, the difference being less than $1 \%$.

Conclusion Although HAL is correlated with height at age 25 for both sexes, and also with arm span and weight at age 25 in females, the percent variance explained by the best fitting model using these parameters is too small to allow for reasonable prediction and inherent implications.

\section{SAT0178 VERTEBRAL DEFORMITIES IN 229 FEMALE RA PATIENTS ASSOCIATIONS WITH CLINICAL AND DEMOGRAPHICAL VARIABLES}

${ }^{1} \mathrm{RE}$ Orstavik, ${ }^{1} \mathrm{G}$ Haugeberg, ${ }^{1} \mathrm{TK}$ Kvien, ${ }^{2} \mathrm{~F}$ Lilleaas, ${ }^{3} \mathrm{~A}$ Hoiseth. ${ }^{1}$ Department of Rheumatology; ${ }^{2}$ Department of Radiology, Diakonhjemmet Hospital; ${ }^{3}$ Department of Rheumatology, Sentrum Institute of Radiology, Oslo, Norway

10.1136/annrheumdis-2001.665

\section{Background}

Objectives To examine the frequency of vertebral deformities in a population based cohort of female rheumatoid arthritis (RA) patients, and their relationship with bone mineral density (BMD) and demographic and clinical variables.

Methods Lateral radiographs of the spine were obtained from 229 female RA patients born 1926 or later (mean age (SD) 63.4 (6.7) yrs, mean disease duration 16.8 (10.4) yrs), recruited from a population based county RA register. Demographic and clinical variables were collected according to standardised procedures. Vertebral deformities were measured using a standardised semiquantitative method described by Genant, by an experienced radiologist $(\mathrm{AH})$. Deformities were classified as mild, moderate or severe (a reduction in any of the vertebral heights of 20$25 \%, 25-40 \%$ and more than $40 \%$, respectively). The patients underwent BMD measurements by DEXA of the femoral neck, total hip and lumbar spine (L2-L4) (Lunar Expert). T-scores were computed as provided from the manufacturer.

Results The total number of deformities was 168 (87 moderate or severe). Forty-nine $(21.4 \%)$ of the patients had at least two mild deformities or one moderate/severe deformity. Mean BMD
(SD) for femoral neck was $0,806 \mathrm{gm} / \mathrm{cm}^{2}$ (0.152), total hip $0.836 \mathrm{gm} / \mathrm{cm}^{2}(0.158)$ and L2-L4 $1,053 \mathrm{gm} / \mathrm{cm}^{2}(0,201)$. In bivariate analysis, vertebral deformities were associated with age, ESR, number of deformed joints, previous non-vertebral fracture, long-term corticosteroid use and with BMD at all three measurement sites ( $p<0.05$ for all variables). In logistic regression analysis, VD was independently associated with age, longterm corticosteroid use and with low bone mass at the hip.

\begin{tabular}{lllll}
\multicolumn{2}{l}{ Abstract SAT0178 Table 1} \\
\hline & $\beta$ & SE & Odds ratio & Confidence interval \\
\hline Age & 0.16 & 0.04 & 1.17 & $1.08-1.28$ \\
Long-term corticosteroid use $^{*}$ & 1.93 & 0.57 & 6.92 & $2.23-21.46$ \\
Previous non-vertebral fracture & 1.34 & 0.46 & 3.82 & $1.55-9.41$ \\
Z-score $\leq-1$ SD total hip & 0.97 & 0.47 & 2.64 & $1.04-6.7$ \\
\hline
\end{tabular}

*12 months or more.

Conclusion This study indicates that several factors are independently related to vertebral deformities in RA, and should be considered when trying to identify patients with this complication. Physicians should probably be especially aware of vertebral deformities in patients with a history of non-vertebral fractures and long-term corticosteroid use.

\section{SAT0179 BACK PAIN AND DISABILITY BEFORE, AND AFTER, CLINICALLY-DIAGNOSED VERTEBRAL FRACTURES}

${ }^{1} \mathrm{M}$ Nevitt, ${ }^{1} \mathrm{~L}$ Palermo, ${ }^{2} \mathrm{D}$ Thompson, ${ }^{1} \mathrm{D}$ Bauer, ${ }^{1} \mathrm{~K}$ Stone, ${ }^{3} \mathrm{~K}$ Ensrud, ${ }^{3} \mathrm{H}$ Fink, ${ }^{1} \mathrm{D}$ Black. ${ }^{1}$ Epidemiology and Biostatistics, University of California, San Francisco; ${ }^{2}$ Merck Scientific Communications, Rahway; ${ }^{3}$ Epidemiology, University of Minnesota, Minneapolis, USA

\subsection{6/annrheumdis-2001.666}

Background Painful vertebral fractures that come to medical attention (CVFx) comprise less than a third of all new vertebral deformities (VDef), yet account for a large proportion of the pain and disability due to vertebral osteoporosis. Little is known about the natural history of back pain and related disability before and after a CVFx.

Objectives We compared prefracture levels of back pain and back disability in women who subsequently experienced a CVFx to levels of back pain and disability in women who did not have a CVFx, and examined the duration of increased back pain and disability for 12 months following a CVFx.

Methods We studied 6,459 women in the Fracture Intervention Trial, aged 55-80 with femoral neck BMD T-score $<-1.6 .33 \%$ had a baseline VDef. CVFx were self-reported community-diagnosed spine fractures confirmed by central comparison of the diagnostic spine films with the study baseline spine films. Back pain and back-related disability (score $0-20$ ) in the past 4 weeks and number of days back disability (bedrest + limited activity) in the previous 12 months were assessed at baseline (prefracture) using validated instruments. Number of days of severe back pain and disability due to back pain were evaluated for successive 3 month intervals before, and after, a CVFx. Baseline and new VDef during 3 to 4.5 yrs of follow-up were assessed with morphometry.

Results Women who had a single new CVFx $>=6$ months after baseline had greater baseline disability than women who had neither a CVFx nor a VDef during FU, adjusting for age, number of baseline VDef, spine BMD, prior $\mathrm{Dx}$ of spine 